\title{
Photo-graphies
}

\section{Anne Cartier-Bresson - La restauration des photographies comme accès à la genèse des œuvres}

Entretien avec Monique Sicard

Monique Sicard

(2) OpenEdition

Journals

Édition électronique

URL : http://journals.openedition.org/genesis/1473

DOI : 10.4000/genesis. 1473

ISSN : 2268-1590

Éditeur :

Presses universitaires de Paris Sorbonne (PUPS), Société internationale de génétique artistique littéraire et scientifique (SIGALES)

\section{Édition imprimée}

Date de publication : 15 avril 2015

Pagination : 89-97

ISBN : 978-2-84050-992-9

ISSN : 1167-5101

Référence électronique

Monique Sicard, « Anne Cartier-Bresson - La restauration des photographies comme accès à la genèse des œuvres », Genesis [En ligne], 40 | 2015, mis en ligne le 28 mars 2017, consulté le 16 mai 2019. URL : http://journals.openedition.org/genesis/1473 ; DOI : 10.4000/genesis.1473 


\title{
Anne Cartier-Bresson \\ La restauration des photographies comme accès à la genèse des œuvres
}

\author{
Entretien avec Monique Sicard
}

Anne Cartier-Bresson est directrice de l'Atelier de restauration et de conservation des photographies de la Ville de Paris (ARCP). À ce titre elle est au cour d'une histoire matérielle de la photographie. Ses travaux et ses recherches la conduisent tant à mieux comprendre les processus de conception et de fabrication des photographies, du XIXe siècle à nos jours, qu'à appliquer des méthodes d'analyse des œuvres spécifiques.

L'ARCP a été créé en 1983. Il a pour mission de mettre en ouvre la politique de préservation du patrimoine photographique municipal. L'équipe compte quatorze personnes réparties au sein de cinq sections : conservation préventive, restauration, reproduction, documentation et régie. Le métier de restaurateur est régenté par les chartes déontologiques émises par l'ICOM-CC (International Council of Museums - Committee for Conservation), l'IIC (International Institute of Conservation) et l' AIC (American Institute of Conservation). Ces codes éthiques s'appliquent aux objets photographiques comme aux autres biens culturels, tout en prenant en compte la spécificité du médium.

L'ARCP participe à la formation des élèves restaurateurs à l'Institut national du patrimoine (INP). Les travaux et mémoires de fin d'études des étudiants de la section Photographie participent depuis 1993 à la connaissance des objets photographiques. L'ARCP et l'INP ont organisé les 17 et 18 novembre 2014 un colloque international pluridisciplinaire sur le thème : "De la matérialité de la photographie à sa dématérialisation : quels enjeux pour la conservation- restauration?».

Monique Sicard - La différence est importante entre la réparation d'une image qui, pour une raison ou une autre, a été abîmée et la restauration d'un objet photographique. Cette dernière n'oblige-t-elle pas à prendre en compte une double histoire : celle de l'objet lui-même, et celle, plus générale, de la photographie? Pouvez-vous, pour résumer, préciser les différences que vous établissez entre image photographique et objet photographique?

Anne Cartier-Bresson - La différence entre un acte de réparation - qui relève d'une pratique artisanale ou purement mécanique - et un acte de restauration méthodologiquement contrôlé est très importante et sera la même pour toutes les spécialités de la conservationrestauration confondues. Il ne s'agit pas de la même intention ni du même but. Un conservateur-restaurateur doit prendre en compte tous les aspects - historiques, matériels, symboliques - de l'objet, que celui-ci soit photographique ou non. Il doit avant tout posséder une connaissance complète du contexte de production de l'œuvre et de sa signification d'origine. Il est soumis à des

1. Colloque organisé à l'occasion des trente ans de l'ARCP et des vingt ans de la spécialité Photographie du département des restaurateurs de l'INP. Pour plus d'informations : <www.inp.fr $>$ et $<w w w . a r c p . p a r i s . f r>$. 
règles déontologiques communes à l'ensemble des biens culturels et son but ultime est de préserver les qualités intrinsèques de l'objet original dans le temps. Il ne s'agit plus alors de faire des améliorations esthétiques ou de masquer des altérations subies, mais de réintégrer une œuvre dans sa fonction d'origine et de lui redonner une stabilité perdue afin de pouvoir mieux la comprendre et la diffuser auprès du public.

La photographie est née en France et cela avant même la contribution de Daguerre et l'invention du daguerréotype. Ainsi, pour appréhender l'histoire matérielle de la photographie, nous disposons de sources que beaucoup nous envient. Nous pouvons regarder et analyser les œuvres originales, mais aussi étudier les documents, manuels ou revues qui s'y rapportent. La fonction de restaurateur oblige à une claire conscience de la terminologie et à une connaissance pointue de l'évolution des pratiques. Pour lui, il n'y a aucune ambivalence des photographies : il ne peut bien sûr confondre image et objet !... Quand j'ai commencé à travailler dans ce domaine au début des années quatrevingt, des responsables de collections confondaient souvent les différents états de tirages. Beaucoup de gens ne faisaient pas la différence entre un contretype et un original ou entre un tirage ancien anonyme quel qu'il soit, et un tirage original d'époque, par exemple. Mais toute photographie est en même temps une image et un objet... une représentation et un matériau; elle est portée par un objet matériel analogique ou numérique... Elle peut aussi rester à l'état d'image virtuelle si elle est produite et stockée seulement sur fichier numérique. Ce sont ces problématiques qui commencent à être traitées par les restaurateurs actuels. Que devonsnous garder ou même sauver afin de suivre les évolutions historiques du médium?

Nous aurions donc perdu progressivement ce sens de l'objet photographique au profit de la simple image, pour laquelle prime la ressemblance indépendamment des supports, des matériaux, des processus de fabrication...

Des années 1890 jusqu'aux années 1980, quand Kodak aux États-Unis, Agfa en Allemagne, Gevaert en Belgique, Ilford en Angleterre, etc. ont commencé à vendre des surfaces sensibles qui pouvaient conserver leurs propriétés suffisamment longtemps pour que le photographe ne soit pas obligé de préparer son support juste avant la prise de vue, une ère nouvelle s'est imposée. Son arrivée a revêtu un caractère brutal, comparable à celui de la rupture numérique contemporaine. Ces supports industriels ont marqué la photographie du $\mathrm{xx}^{\mathrm{e}}$ siècle, qui a perdu en intention artistique pour devenir une information, un moyen, une application. Ce fait a favorisé la multiplication des genres : photographie de mode, de publicité, etc. À partir de là, les bibliothèques et les musées se sont désintéressés de l'objet photographique. La confusion des genres s'est installée. Le souci de dater, d'authentifier et de caractériser les images s'est perdu. Tout cela a conduit à cette photographie du $\mathrm{XX}^{\mathrm{e}}$ siècle dont les fonds ont été mal conservés, pillés, abandonnés.

L'information portée par l'image a pris le relais de cet objet qui n'était plus porteur d'une attention particulière. On estimait alors que restaurer, c'était rephotographier. L'acte de restauration était confondu avec celui du photographe qui faisait une « restitution optique ». On cherchait en outre à améliorer cette copie en réalisant des traitements chimiques afin d'ôter le voile formé sur les daguerréotypes, de faire ressortir des détails, etc., avec l'idée qu'il convenait non pas de reconstituer la photographie primitive, mais de retrouver l'information dont elle était porteuse. Cette seconde photographie n'était pas superposable à l'original. L'objet historique lui-même, son intégrité, n'avaient plus d'importance ; ils n'étaient pas respectés.

On peut citer l'exemple d'un tirage d'André Vigneau (1892-1968, photographe, cinéaste, peintre et sculpteur français), sur lequel du feutre indélébile a été apposé pour souligner certains détails de l'image. Nous ne savons aujourd'hui ni par qui, ni quand. S'agit-il d'un vandalisme ou est-ce de la main de l'artiste ? Il faut aujourd'hui se renseigner sur Vigneau, savoir de quelle époque date le feutre, reconstituer les faits de genèse, regarder les archives et lire les documents historiques qui se rapportent à cette image. Nous héritons de cette période de production photographique qui s'étend sur plus d'un siècle et durant laquelle ont été utilisés des matériaux qui n'ont jamais été catalogués, inventoriés ou caractérisés correctement. 
Au tournant des $\mathrm{XIX}^{\mathrm{e}}$ et $\mathrm{XX}^{\mathrm{e}}$ siècles, la restauration des peintures était, elle, déjà prise en charge par des professionnels. Les restaurateurs étaient déjà distincts des créateurs, même s'il existait encore des ateliers où les peintres restauraient eux-mêmes leurs peintures, la profession n'étant pas encore réglementée. La restauration scientifique des peintures et des sculptures a été mise en place au $\mathrm{Xx}^{\mathrm{e}}$ siècle avec la naissance des laboratoires des grands musées européens et américains. Mais jusqu'à une époque récente, la photographie n'était pas perçue comme une œuvre patrimoniale. Or, la prise en compte d'un objet photographique façonné, fabriqué et historiquement daté implique l'existence de collectionneurs, le développement d'un marché de la photographie. À l'époque où la photographie originale n'avait qu'une valeur d'information, sans valeur économique intrinsèque, des fonds entiers de négatifs ont été jetés, une fois le tirage effectué.

Cette période semble heureusement terminée. Les archives photographiques sont aujourd' hui étudiées, valorisées. Le marché de la photographie d'art, né dans les années 1970-1980, se porte plutôt bien. Le concept d'objet photographique occuperait-il de nouveau le devant de la scène? Pourtant, l'histoire ne se répète pas. Les expérimentateurs des premiers temps auraient-ils laissé place à d'autres spécialistes de l'objet photographique : les collectionneurs, mais aussi, certainement, les restaurateurs?

Les collectionneurs ont toujours existé ; c'est la forme et la manière de collectionner qui a changé. Pour le restaurateur, c'est une autre problématique ; il travaille désormais dans un contexte professionnel qui est celui de la spécialisation à l'intérieur des métiers de la conservation-restauration des biens culturels. La discipline n'a pu émerger que lorsque les photographies ont acquis une double valeur, sur le plan patrimonial et sur celui du marché de l'art. Dès lors, la nécessité d'une formation professionnelle, tant pratique que théorique, spécifique aux restaurateurs de photographies fut reconnue ; le restaurateur est devenu un spécialiste de l'action préventive et curative et surtout il a appris à identifier les objets et à en comprendre le contexte historique de production, ainsi que sa valeur d'usage. Cela se produisit à la toute fin des années 1970, d'abord aux États-Unis, puis en Angleterre, en France et dans quelques pays pionniers tels que le Danemark.

L'acte du restaurateur est avant tout soumis à la compréhension des matériaux historiques en présence et à leurs processus d'altération. Comme dans les autres domaines, il faut insister sur le fait que l'on ne restaure pas ce que l'on ne comprend pas, autant sur le plan matériel que sur le plan de l'histoire.

Prenons l'exemple de Tourelle près de l'Hôtel de Ville (fig. 1). Ce daguerréotype anonyme est conservé à la Bibliothèque historique de la Ville de Paris. Il représente un immeuble qui a été détruit depuis. Il s'agit d'une pleine plaque, dont le format, $21 \times 16 \mathrm{~cm}$, est celui de la chambre photographique de Daguerre. Cet objet, assez exceptionnel du point de vue technologique, et bien qu'anonyme, possède une grande valeur historique et occupe une place importante dans l'histoire de la photographie. Malheureusement, dans les années 19501960, un responsable des collections a dû se dire qu'il fallait préserver cette plaque remarquable. Le fixé sous verre, qui protège le daguerréotype de l'oxydation de l'air, était cassé ; un voile d'oxydation s'était donc déposé à l'endroit de la cassure. Il a cru bien faire en inversant la position du fixé sous verre. Si l'intention peut sembler louable, il a agi sans connaissance des phénomènes en jeu, et a créé un nouveau front d'oxydation, beaucoup plus dommageable que le premier, illustrant ainsi l'importance de la compréhension des mécanismes d'altération dans les actions de conservation préventive et de l'analyse « archéologique » que le restaurateur doit porter sur un objet altéré.

Pour le restaurateur, authentifier l'objet revient aussi à vérifier qu'il ne s'agit pas d'une copie, à préciser sa fonction et son usage. Un tirage original n'est pas à confondre avec un tirage posthume, ou une photographie de presse avec un tirage d'exposition. Authentifier consiste à comprendre l'objet sur un plan fonctionnel, historique et économique, mais non sur ce seul dernier critère qui est un paramètre propre au marché de l'art. Le restaurateur n'est pas un marchand. Il n'est pas non plus un historien au sens strict, mais il doit s'appuyer sur l'histoire pour comprendre le contexte de création de l'œuvre en présence. Le restaurateur n'est 

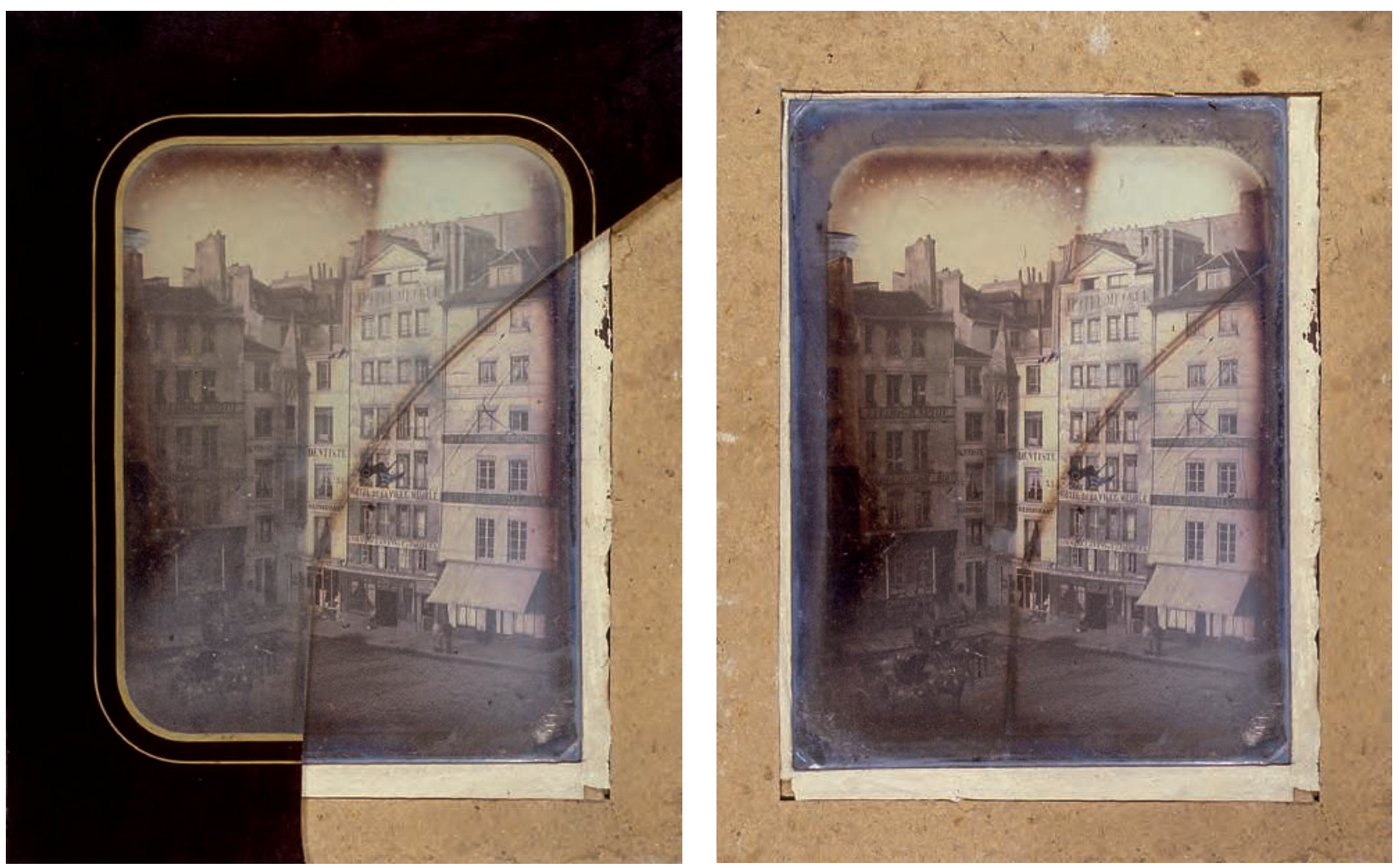

Fig. 1 : Anonyme, Tourelle près de l'Hôtel de Ville (daguerréotype, 1840-1850, 4-EPE-002) À gauche : plaque avec fixé sous verre cassé, inversé / À droite : plaque nue après dégagement du fixé sous verre cassé, montrant les deux fronts d'oxydation successifs

(C) Bibliothèque historique de la Ville de Paris/ARCP/Mairie de Paris

pas un scientifique mais il prend appui sur les sciences expérimentales : il a une bonne connaissance des matériaux, des processus d'altération, des mécanismes physico-chimiques en jeu dans ses restaurations.

On le définit souvent en creux, par ce qu'il n'est pas, mais il peut être défini plus positivement : le restaurateur est un spécialiste des techniques, connaisseur des processus d'altération et susceptible de se renseigner auprès d'autres experts. Il gère la double compatibilité des matériaux et de l'original qu'il met en contact : sur le plan physico-chimique et sur le plan historique. Il agit de manière pluridisciplinaire, s'appuyant sur des connaissances parallèles afin d'avoir une compréhension globale de l'objet avant d'intervenir. Ses responsabilités sont importantes car il agit directement sur le patrimoine.

Pourriez-vous préciser, à l'aide d'un exemple, quels sont les apports de la restauration, de ses pratiques, de ses savoirs, à la connaissance de l'objet photographique et des processus de sa création?

Dans le cadre de son intervention, le restaurateur peut être amené à procéder au démontage de photographies enchâssées telles que les daguerréotypes et les ambrotypes par exemple. Cet acte permet d'accéder exceptionnellement à des informations relatives à leur mode de fabrication. En effet, l'extrême fragilité des daguerréotypes rend risquée toute opération de démontage, qui ne peut être menée que par un spécialiste. Cette intervention n'est d'ailleurs jamais effectuée pour un simple examen mais uniquement lorsque la nécessité d'une restauration le justifie.

Nous pouvons prendre l'exemple d'un portrait d'homme issu de la collection de l'Agence Roger-Viollet, de format quart de plaque, signé Ollivier et datant de 1847 (fig. 2). La mise à nu de la plaque a révélé le mode d'argenture - par laminage sans couche additionnelle appliquée par galvanoplastie -, ainsi que les poinçons figurant sur le bord de la plaque - plaque Sheffield et titre d'argent trente millièmes. L'exposition de la plaque sous rayonnement ultraviolet de longueur d'onde spécifique a mis en évidence la présence de complexes cyanhydriques, ce qui suggère l'utilisation de composés à base de cyanure, soit au cours de la fabrication de l'image daguerrienne, lors de l'étape de fixage ou de virage, soit à l'occasion d'un nettoyage dans le cadre d'une restauration réalisée au XIX'e siècle (fig. 3 à 5). 

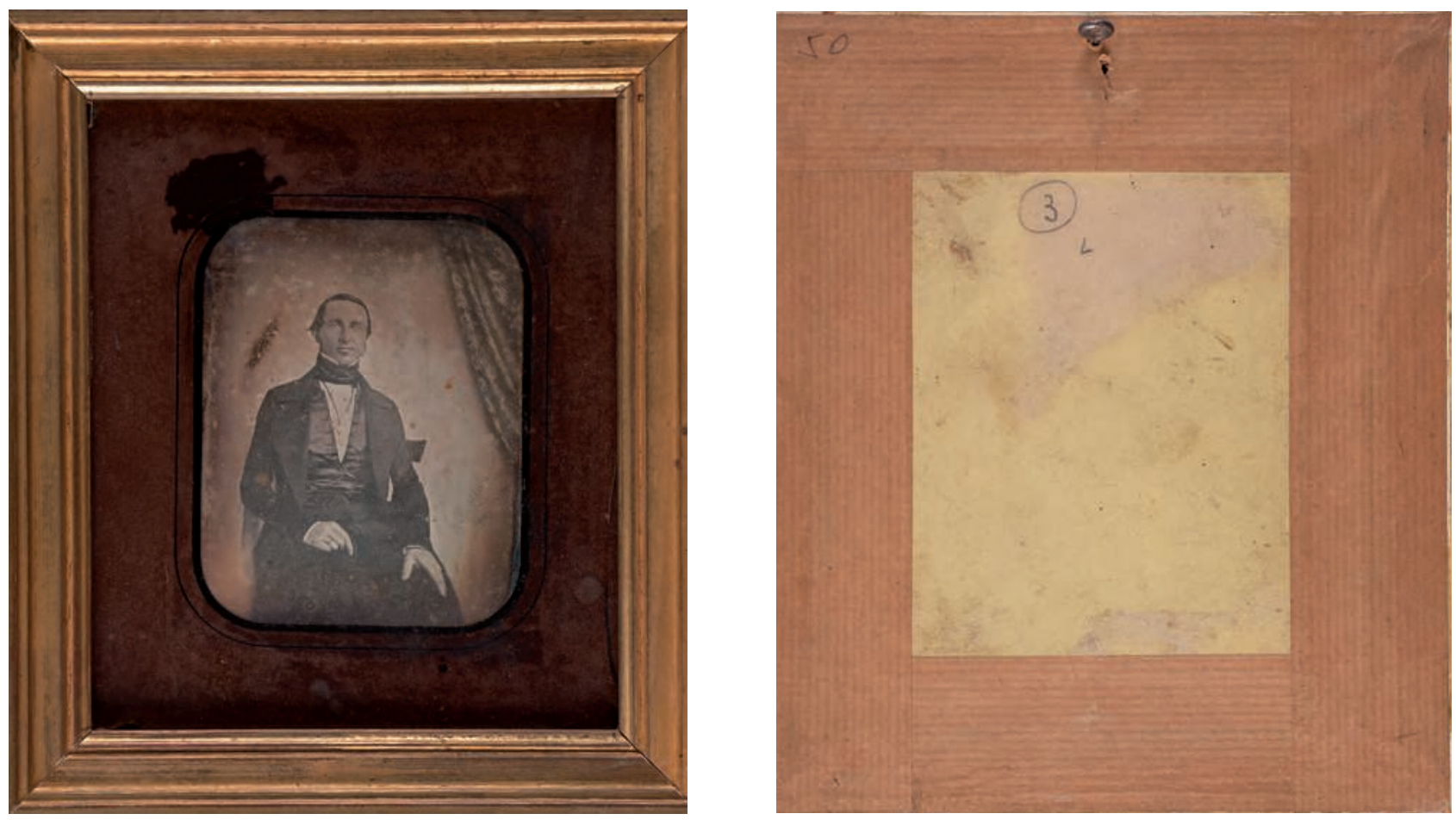

Fig. 2 : Ollivier, Portrait d'un homme, «Ollivier 1847 »(daguerréotype, 1847, collections Agence Roger-Viollet) À gauche : recto de l'œuvre avant démontage / À droite : verso avant démontage (C) Reproduction : ARCP/Mairie de Paris
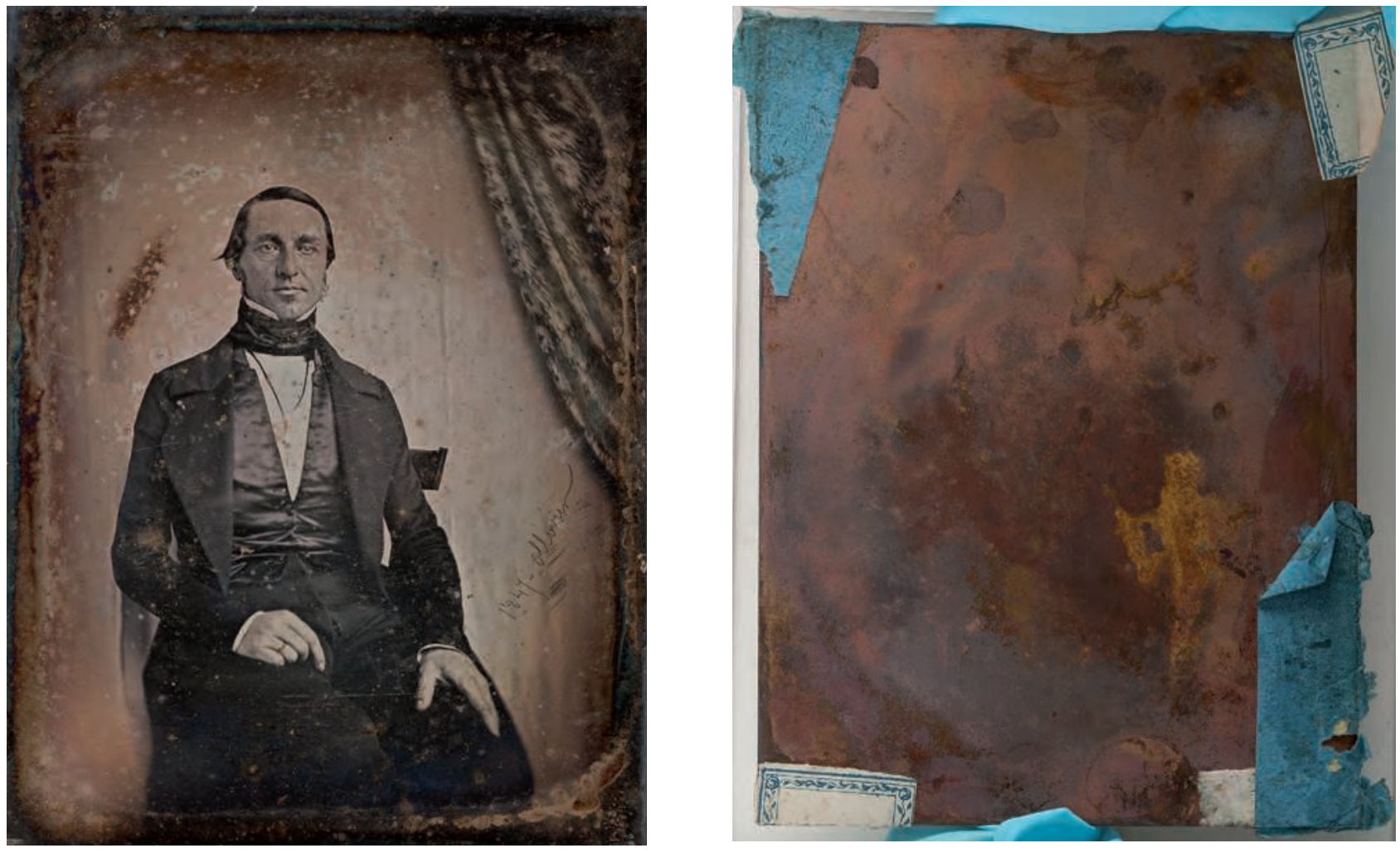

Fig. 3 : Plaque après démontage, recto et verso. Prise de vue en lumière réfléchie (c) Reproduction : ARCP/Mairie de Paris 

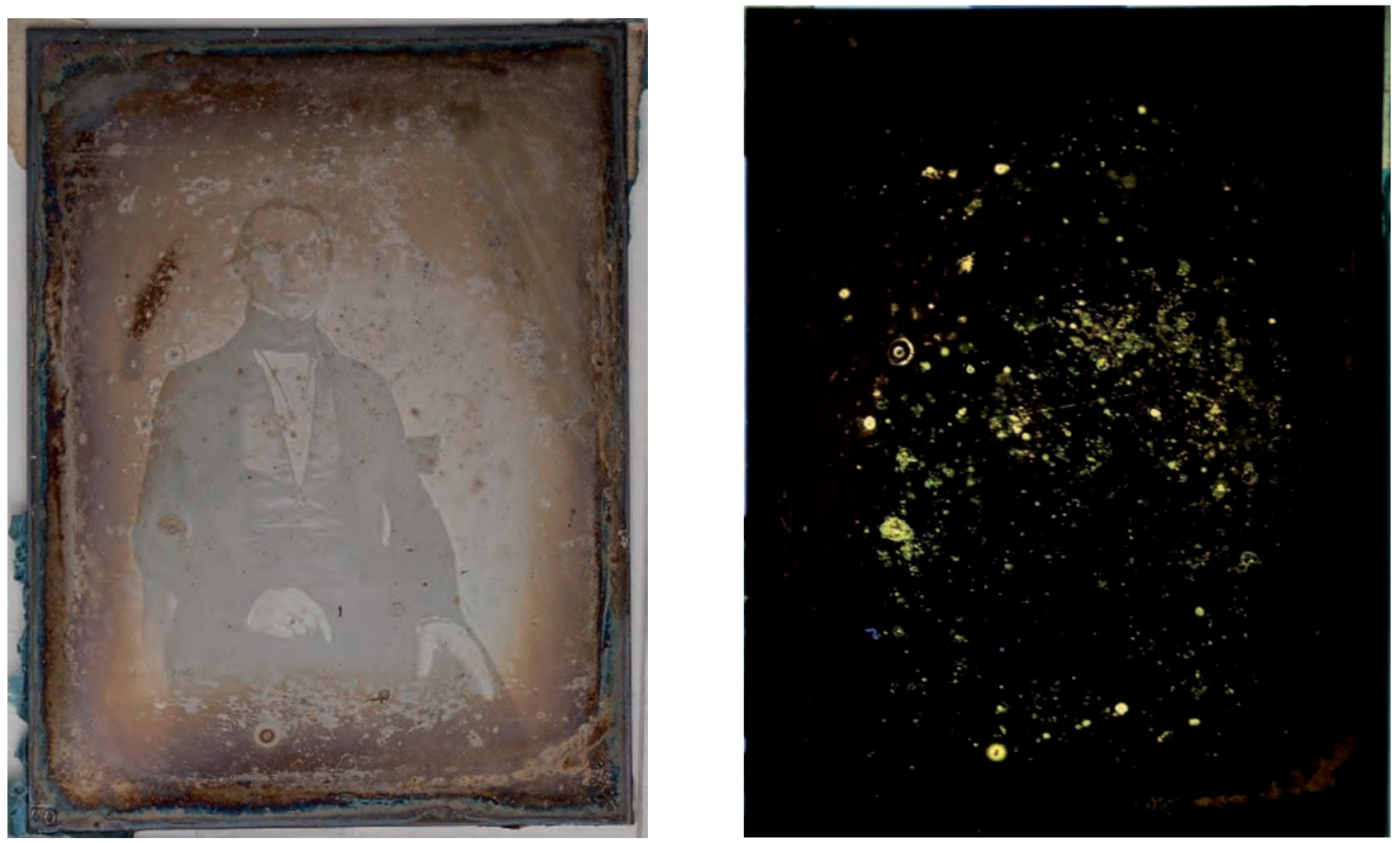

Fig. 4 : Plaque après démontage, recto

À gauche : prise de vue en lumière spéculaire / $\grave{A}$ droite : prise de vue en lumière UVC

(C) Reproduction : ARCP/Mairie de Paris
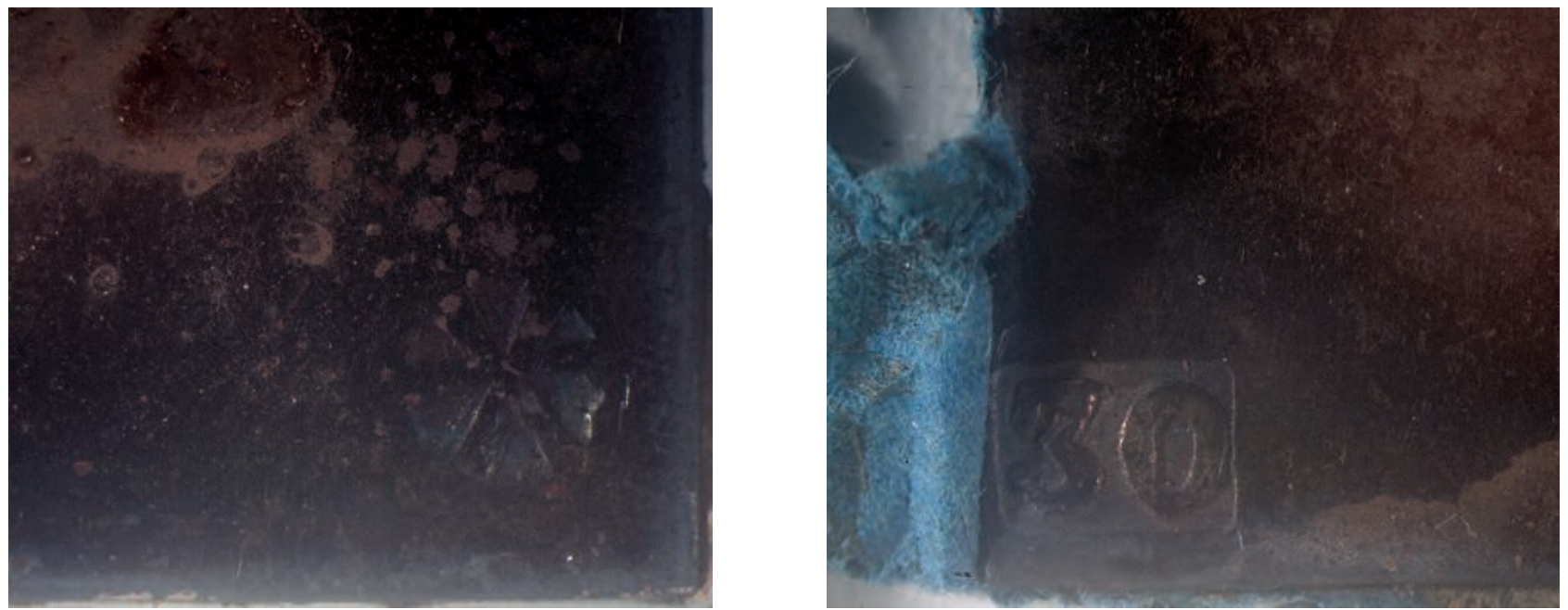

Fig. 5 : Poinçons figurant sur la plaque

(C) Reproduction : ARCP/Mairie de Paris 
Toutes les informations recueillies à l'occasion d'un démontage sont précieuses. Le projet européen Daguerreobase, mis en œuvre depuis 2012, a pour objet de répertorier ce type de données au sein d'une base collective accessible en ligne. Dix-huit institutions de treize pays européens sont chargées de l'alimenter, avec pour objectif cible le recensement et la description fine de vingt-cinq mille daguerréotypes conservés dans les institutions publiques et privées $^{2}$.

Une autre restauration récente réalisée à l'ARCP sur une œuvre de Michel Journiac, L'Inceste, datant de 1974 et conservée à la Maison européenne de la photographie, peut aussi être prise pour exemple : ce photomontage de silhouettes découpées et collées sur un support secondaire daté et signé, présentait une altération évolutive due à la colle utilisée pour sa réalisation. Afin de retirer ces résidus de colle, l'œuvre a dû être démontée du support secondaire. Lors de cette opération, il a été découvert au revers des supports primaires des photographies de nombreuses traces d'arrachage ainsi que des éléments résiduels d'un précédent support secondaire, indiquant que l'œuvre avait été démontée au moins deux fois, ce qui pose au conservateur et au restaurateur la question de l'interprétation des phénomènes mis en lumière.

Vous venez d'évoquer l'apport des techniques, réflexions et savoirs de la restauration à la connaissance de l'objet photographique et de son histoire. Mais quels seraient les apports spécifiques d'une conservation dite "préventive " à la connaissance de l'objet et de ses processus de création?

Le rôle de la conservation préventive permettra de mieux faire connaître un objet à l'historien des temps futurs. Il consiste à étudier et établir des bilans généraux et des diagnostics particuliers afin de préserver globalement les collections. Cette facette de la restauration lui est indissociable et s'appuie bien évidemment sur une connaissance précise et très spécifique des divers contextes. La prévention qui permet néanmoins d'éviter les altérations et donc les besoins de restauration dans le temps permettra de laisser aux générations futures des objets authentiques et intacts, afin d'apprécier l'histoire de la photographie autrement que par les reproductions dans les livres! Par ses méthodes et les actions nécessaires qu'elle implique, la conservation préventive contribue néanmoins à la connaissance des fonds. Elle intervient à toutes les étapes de la vie d'une photographie : au sein même des expositions ou des aires de stockage, mais aussi lors de sa numérisation ou de sa communication au public. Pour les expositions itinérantes par exemple, nous travaillons de manière très fine sur les œuvres. Ainsi, nous avons réalisé toute une étude des craquelures dans les couches d'albumine de certaines photographies d'Atget (fig. 6). Elles ont été suivies dans le temps sous monitoring afin de déterminer leur comportement au cours des itinérances de l'exposition.

La conservation préventive, qui s'appuie sur des bilans de conservation et des études spécifiques, apporte de facto des éléments de datation, d'authentification et d'analyse des œuvres avant leur utilisation par le biais par exemple de l'exposition ou de la numérisation (fig. 7).

Prendre en compte l'objet photographique vise à retracer son histoire singulière. Tout objet photographique serait donc différent d'un autre objet. Les restaurateurs modernes se feraient-ils les chantres d'une unicité retrouvée?

Pour que la restauration photographique devienne un métier à part entière, avec ses spécificités intellectuelles, historiques, culturelles et manuelles, il a fallu, comme nous l'avons vu précédemment, que l'on s'intéresse à l'objet photographique et non plus à la seule image. Dans les années 1980-1990, nous devions faire valoir cette unicité de l'œuvre. Le combat a été partiellement gagné au sein des musées, des bibliothèques spécialisées, des milieux du patrimoine mais pas toujours auprès des archives, des agences photographiques ou des professionnels de la photographie.

Dans le cadre de l'enseignement de la discipline, les étudiants apprennent à faire des constats d'état et des études de collection globales ou individuelles. Par les dossiers d'œuvres, ils identifient individuellement les objets photographiques, en les rapportant au corpus général auquel ils appartiennent.

2. <www.daguerreobase.org>. 

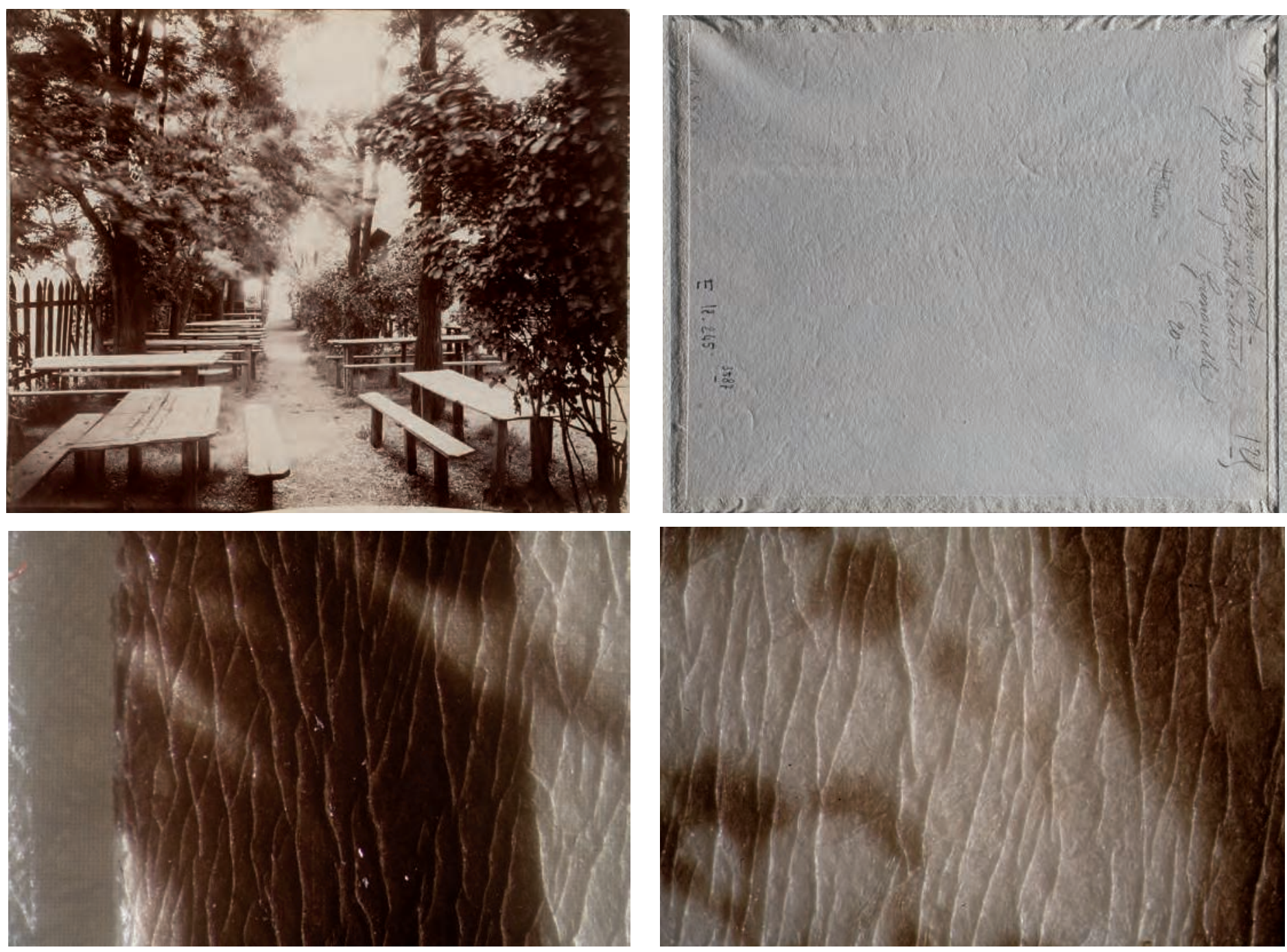

Fig. 6 : Reproductions réalisées par l'ARCP dans le cadre d'une étude des craquelures dans les couches d'albumine de photographies d'Eugène Atget En haut à gauche : Eugène Atget, Fortifications, porte de Ménilmontant, Paris (XXe arr.), vers 1910 (tirage sur papier albuminé, 1905-1915) / En haut à droite : recto de l'œuvre, prise de vue en lumière rasante En bas : recto de l'œuvre, détails agrandis

(c) Eugène Atget/Musée Carnavalet/ARCP/Mairie de Paris - Reproduction ARCP/Mairie de Paris 


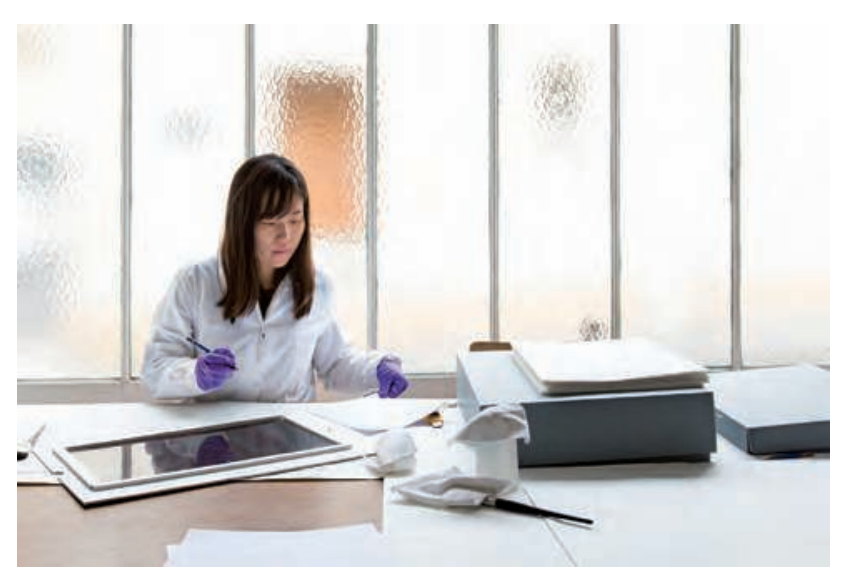

Fig. 7 : Étude de conservation d'un fonds de négatifs sur verre des collections de l'Agence Roger-Viollet, 2014 (C) Estelle Poulalion

Sachant que l'auteur de la photographie est celui qui a réalisé la prise de vue, il n'en reste pas moins que le tireur - qui peut être ou non l'auteur de la prise de vue - joue un rôle important dans le résultat final. Il est essentiel de décrire les œuvres de manière complète et correcte, dans les catalogues, les expositions, les inventaires et lorsque les images sont numérisées, avec ce type de mention également. Il ne suffit plus de dire «Fox Talbot, buste de Patrocle, papier salé » ou «Anna Atkins, cyanotype ». Il faut insérer l'objet dans des corpus, dans une histoire. Anna Atkins a réalisé des cyanotypes en 1844 . Elle a produit ses photogrammes en posant directement des algues ou des champignons sur un papier aux sels de fer sensible à la lumière, comme l'indique la couleur bleue. Elle n'a pas utilisé d'appareil photographique. Cela signifie que ce cyanotype est un négatif, et donc un objet unique. Elle aurait pu rendre son photogramme translucide avec de la cire, puis l'utiliser comme matrice pour multiplier l'image, mais cela n'était pas du tout sa problématique. Elle faisait œuvre de connaissance scientifique, non de diffusion. D'autre part, il est important de connaître la source, la matrice de l'image positive. Cela devient encore plus important avec les images numériques car les variétés de tirages et d'impressions numériques qui circulent sur le marché sont nombreuses. Ce n'est pas parce que l'on se situe dans le monde contemporain et dans les technologies numériques que l'on peut être plus laxiste dans la compréhension des dispositifs et dans la caractérisation des fonds, bien au contraire. L'image est-elle issue d'un négatif analogique qui a été scanné ou d'une matrice numérique? Le tirage final est-il analogique ou numérique ? Quelle transformation l'auteur a-t-il apportée ? Quelle a été son intention?

Les œuvres possèdent chacune une esthétique et une fragilité qui leur sont propres. Si elles ne sont pas caractérisées, précisément légendées, nul ne pourra jamais ni les restaurer, ni les conserver. C'est pourquoi nous avons développé à l'ARCP, en partenariat avec nos collègues de l'AIC, un questionnaire que les artistes sont invités à remplir avant toute acquisition de photographie par les institutions municipales ${ }^{3}$. Si l'image photographique peut être multiple, l'objet photographique, lui, est toujours unique.

Il faut cesser de réduire la photographie à un système « négatif, positif, multiple » car tous les objets photographiques sont datés et par là uniques. Ils sont chacun riches de leur propre histoire, fussent-ils des fichiers numériques.

\section{Références bibliographiques}

CARTIER-Bresson Anne, « La photographie contemporaine sous le regard de la conservation et de la restauration : le cas du "Plan couleur et impressions numériques" de la Ville de Paris », Technè, $\mathrm{n}^{\circ}$ 37, 2013, p. 49-60.

-, Dans l'atelier du photographe, la photographie mise en scène, Paris, Paris-Musées, 2012.

Cartier-Bresson Anne (dir.), Le Vocabulaire technique de la photographie, Paris, Marval/Paris-Musées, 2008.

Cartier-Bresson Anne, Ploye Françoise, L'Objet photographique, une invention permanente, Arles, Actes Sud, coll. « Photo Poche », 2012.

3. «Formulaire d'information sur les photographies », adaptation par l'ARCP du Photographic Information Record, questionnaire international validé par 1'American Institute of Conservation. Ce questionnaire est disponible sur le site de l'ARCP, <www.arcp.paris.fr>. 